\title{
Prenatal Risk Factors for Neurogenic Bladder
}

\author{
Parsa Yousefichaijan, ${ }^{1,}{ }^{*}$ Elham Madandar, ${ }^{2}$ and Mohammad Rafiei ${ }^{3}$ \\ ${ }^{1}$ Department of Pediatric Nephrology, Amirkabir Hospital, School of Medicine, Arak University of Medical Sciences, Arak, Iran \\ ${ }^{2}$ Students Research Committee, Arak University of Medical Sciences, Arak, Iran \\ ${ }^{3}$ Department of Biostatistics, School of Medicine, Arak University of Medical Sciences, Arak, Iran \\ "Corresponding author: Parsa Yousefichaijan, Department of Pediatric Nephrology, Amirkabir Hospital, School of Medicine, Arak University of Medical Sciences, Arak, Iran. Tel: \\ +98-9111357114, E-mail: parsayousefichaijan@yahoo.com
}

Received 2017 July 24; Revised 2017 January 29; Accepted 2017 February 27.

\begin{abstract}
Background: Neurogenic bladder is a term referred to many urinary conditions in persons with uncontrolled state of bladder due to brain, spinal cord, or nerve disorder. Children with congenital anomalies of the spinal cord, such as spina bifida, may also have neurogenic bladder.

objectives: The study aimed to find a possible link between neurogenic bladder and prenatal risk factors.

Methods: In this case-control study, 60 infants with neurogenic bladder and 60 infants without neurogenic bladder, who referred to the Amir-Kabir hospital, were evaluated. Both groups completed preset questionnaires. Maternal information was assessed, and statistical analysis was done by SPSS version 19

Results: The results showed that male gender, maternal age $>35$ years, family history of kidney disease, low education level of parents, low family income, low birth weight (LBW), smoke exposure, chronic hypertension in mothers, gestational hypertension, chronic diabetes mellitus in mothers, gestational diabetes mellitus, maternal BMI $<18.5 \mathrm{~kg} / \mathrm{m}^{2}$ (underweight), and preterm delivery were prenatal risk factors for infantile neurogenic bladder.

Conclusions: The data suggest that prenatal factors may affect the risk of neurogenic bladder. Adequate prenatal care and good maternal support can be effective in the prevention of neurogenic bladder.
\end{abstract}

Keywords: Neurogenic Bladder, Risk Factors, Prenatal

\section{Background}

Bladder dysfunction, also referred to dysfunctional voiding, is a common term to describe both filling and emptying of the vesicles (1). Inefficient urination is a typical clinical issue that is observed in about $40 \%$ of patients referred to pediatric urologists (2). Neuropathic bladder malfunction in children generally may be congenital that is displayed because of neural tube deficiency or other spinal abnormalities (3). The most important urologic consequences of neuropathic bladder dysfunction are urinary incontinence (frequently happening around 4 years of age that is adapted to the individual child), urinary tract infections, and hydronephrosis from vesicoureteral reflux (3). Bladder dysfunction results from disruptions of the normal voiding process by any alteration in the innervation of the bladder or external sphincter, bladder compliance or volume capacity, detrusor muscle function, or structure of the bladder or bladder outlet. These include neurogenic, anatomic, or functional causes. Neurogenic causes of bladder dysfunction disrupt the innervation of the bladder or external sphincter. Neurogenic causes are due to either congenital anomalies such as myelomeningocele or trauma to the central nervous system (e.g., spinal cord injury). Every child with a suspected neurologic abnormality should be evaluated for occult neurologic lesion (4). This study tries to investigate the relationship between infantile neurogenic bladder and prenatal risk factors, hypothesizing that these parental conditions would increase the risk of infantile neurogenic bladder-related renal injury. This is the first study to assess the relationship between maternal factors and neurogenic bladder.

\section{Objectives}

This study tries to investigate the relationship between neurogenic bladder and prenatal risk factors, hypothesizing that these prenatal conditions would increase the risk of neurogenic bladder. This is the first study to assess the relationship between neurogenic bladder and prenatal risk factors.

\section{Methods}

We conducted a population-based case-control study on 60 patients with neurogenic bladder (control group) and 60 infants without neurogenic bladder, who referred to Amir Kabir hospital. Both groups completed preset 
questionnaires. All infants with repeated urinary tract infections (UTI), frequent urination, and symptoms of urinary retention and obstructive bladder were enrolled. A sonogram of the bladder was performed to evaluate bladder anatomy. The participants who did not match the prerequisites in the pretest or those who suffered from extensive comorbidities or were unavailable for the follow-up confounded the study results of the treatment; thus, they were excluded from the sample of the study. Moreover, those participants who had been unresponsive to previous interventions or had experienced another clinical study were excluded from the study since including them would compromise the internal validity of the findings. We evaluated maternal characteristics of the patients and used logistic regression analysis to measure the relationship between prenatal risk factors and neurogenic bladder. Maternal characteristics of the infants with and without neurogenic bladder were as follows: 1 ) age $<18,18$ to 24,25 to 29,30 to 34 , and $>35$ years, 2 ) education level (grade 1 to 12, bachelor's degree), 3) residence in urban or rural areas, 4) smoke exposure, 5) previous pregnancy $(0,1,2,3$, or $>3$ times), 6) prenatal care (inadequate or adequate), 7) chronic and gestational hypertension, 8) maternal diabetes mellitus (gestational diabetes mellitus and chronic diabetes mellitus), 9) maternal body mass index $\left(\mathrm{kg} / \mathrm{m}^{2}\right)(<$ 18.5, 18.5 - 24.9, $25-29.9$, or $>30$ ) and, 10) family history of kidney disease. Infant characteristics were: 1 ) age at diagnosis of study definition of neurogenic bladder $(<1,1-3$, or $>3$ years), 2) birth weight (VLBW $<1500 \mathrm{~g}$, LBW of 1500 $-2500 \mathrm{~g}$, normal BW of $2500-4000 \mathrm{~g}$ and HBW $>4000 \mathrm{~g}$ ), 3) gestational week ( $<37$ as preterm, 37 - 42 term, or $>42$ as post term), 4) sex (male or female) and, 5) type of infant feeding. Statistical analysis was conducted by SPSS version 19.

\section{Results}

Most of the infants were below 1 years of age at the time of neurogenic bladder diagnosis. There was a high prevalence of male children in total patients. Agreater proportion of cases had LBW and gestational age $<37$ weeks when compared to controls. The distribution of single fetus versus multiple births was the same in the case and control groups. Maternal characteristics including education level, urban/rural residence, prior pregnancy and multiple births, age at diagnosis of neurogenic bladder, sex, gestational hypertension, smoke exposure, overweight, obesity, and prenatal care were similar in the case and control groups. The cases more frequently had mothers aged $>35$ years $(P$ value $=0.006)$, chronic and gestational hypertension $(\mathrm{P}$ value $=0.008)$, preterm delivery $(\mathrm{P}$ value $=0.007)$, history of LBW $(\mathrm{P}$ value $=0.0001)$ and $\mathrm{BMI}<18.5 \mathrm{~kg} / \mathrm{m}^{2}$ (underweight) $(\mathrm{P}$ value $=0.031)$, gestational and chronic diabetes mellitus ( $\mathrm{P}$ value $=0.006$ ), family history of kidney disease $(\mathrm{P}$ value $=0.005)$, low family income $(\mathrm{P}$ value $=0.004)$, parental low education level $(P$ value $=0.0001)$, and smoke exposure $(\mathrm{P}$ value $=0.0002)$ compared to controls (Table 1).

\section{Discussion}

In this population-based, case- control study, we showed a relationship between neurogenic bladder and prenatal exposure to gestational HTN, chronic HTN, preterm delivery, LBW, gestational and chronic DM, maternal age over 35 years, and underweight. We found that better diabetes mellitus care in pregnant women could lower the risk of anomalies in infants. Maternal underweight was also found to be associated with infantile neurogenic bladder. Moreover, low birth weight was associated with the development of neurogenic bladder in infants. This suggests that the risk of developing neurogenic bladder in infants may be extensively specified in the uterus and it depends on parental factors.

Yousefichaijan concluded that age more than 35 years, pre-gestational hypertension, preeclampsia and eclampsia, preterm delivery, very low birth weight(VLBW), pre gestational diabetes mellitus, and maternal BMI $<18.5 \mathrm{~kg} / \mathrm{m}^{2}$ (underweight) were prenatal risk factors for infantile reflux nephropathy. The data suggest that prenatal factors may affect the risk of infantile reflux nephropathy. Adequate prenatal care and good maternal support can be effective in the prevention of reflux-related renal injury (5).

Yousefichaijan also indicated that the most significant causes of the mentioned pediatric CKD were urinary tract infection (39.5\%) and growth failure (12.9\%). When the effect of confounding variables were controlled, it was realized that household income, using packed water for drinking, percentile of BMI, and gestational age were the significant predictors of pediatrics $\mathrm{CKD}(\mathrm{P}<0.05)$. Urinary tract infection (UTI) and fever were the most common signs of the disease. For children, the leading cause of CKD was reflux nephropathy (6).

Sokal demonstrated that a greater proportion of cases with congenital anomaly (CA) were in males and the risk of any CA was $26 \%$ greater in males. Our PRs were highly compatible with those of previous studies. It seems that sociodemographic and parental factors do not affect these risks (7).

Tain showed that the incidence of congenital anomalies of the kidney and urinary tract (CAKUT) was nearly 4.2 per 10,000 births. Maternal age of 20 to 29 , or 30 to 39 , maternal gestational diabetes, maternal thalassemia/hemochromatosis, polyhydramnios or oligohy- 
Table 1. Maternal Characteristics of Study Group

\begin{tabular}{|c|c|c|c|}
\hline Maternal Characteristics & Case & Control & P Value \\
\hline Maternal BMI $\left(\mathrm{kg} / \mathrm{m}^{2}\right)<18.5$ & $25(20.8 \%)$ & $9(7.5 \%)$ & 0.031 \\
\hline Chronic diabetic mellitus & $25(20.8 \%)$ & $4(3.3 \%)$ & 0.006 \\
\hline Gestational diabetic mellitus & $33(27.5 \%)$ & $11(9.2 \%)$ & 0.006 \\
\hline Chronic hypertension & $40(33.3 \%)$ & $13(10.8 \%)$ & 0.008 \\
\hline gestational hypertension & $28(23.3 \%)$ & $6(5 \%)$ & 0.008 \\
\hline Mother age $>35$ years old & $82(68.3 \%)$ & $5(4.1 \%)$ & 0.006 \\
\hline Gestational age $<37$ weeks & $80(66.7 \%)$ & $21(17.5 \%)$ & 0.007 \\
\hline History of low birth weight (neonatal birth weight $1500-2500 \mathrm{~g}$ ) & $62(51.7 \%)$ & $19(15 \%)$ & 0.0001 \\
\hline Smoke exposure & $82(68.3 \%)$ & $36(30 \%)$ & 0.0002 \\
\hline
\end{tabular}

dramnios, prior pregnancy more than 1 times, having gestational age $<37$ weeks, and being a boy were prenatal risk factors for congenital anomalies of the kidney and urinary tract. The infants of those mothers who are diagnosed with gestational diabetes were more prone to congenital anomalies, small gestational age ( $<37$ weeks), and low birth weight. CAKUT were associated with some of the maternal health risk factors (8).

Raisanen concluded that smoking cessation could reduce pregnancy risks. Exposure to smoking in early pregnancy could increase admission to neonatal intensive care unit (NICU) (9).

Sadiq suggested that the typical cause of occult spinal dysraphism in children up to the age of 15 years old is neurogenic bladder. Besides, more than $60 \%$ of the cases diagnosed with neurogenic bladder had myelomeningocele, meningocele, and sacral agenesis (10).

In subordinate group examination with chronic kidney disease (CKD), low birth weight (LBW $<2500 \mathrm{~g}$ ) and maternal chronic DM were meaningfully associated with the risk of kidney injury. In addition, low birth weight, maternal gestational DM, and maternal obesity were significantly correlated with obstructive urinary tract disease. The data suggest that there are some factors acting before birth that may affect the risk of chronic kidney disease (11).

Bauer concluded that neuropathic bladder takes place in patients in a wide range of conditions such as congenital conditions. These conditions have a particular relationship with anatomic anomalies and procured conditions caused by accident-related injuries (12).

We think that our article needs a larger study to evaluate neonates with improved neurogenic bladder. Low birth weight, maternal gestational DM, and maternal overweight/obesity had significant relationships with obstructive uropathy.

\section{Acknowledgments}

The authors gratefully acknowledge the research council of Arak University of Medical Sciences (grant number: 2341) for the financial support.

\section{Footnote}

Financial Disclosure: This study was financially supported by Arak University of Medical Sciences.

\section{References}

1. Farhat W, Bagli DJ, Capolicchio G, O'Reilly S, Merguerian PA, Khoury A, et al. The dysfunctional voiding scoring system: quantitative standardization of dysfunctional voiding symptoms in children. J Urol. 2000;164(3 Pt 2):1011-5. [PubMed: 10958730].

2. Feldman AS, Bauer SB. Diagnosis and management of dysfunctional voiding. Curr Opin Pediatr. 2006;18(2):139-47. doi: 10.1097/01.mop.0000193289.64151.49. [PubMed: 16601493].

3. Robert MK, Bonita FS, Joseph WG, Nina FS. Nelson Textbook of Pediatrics. 20 ed. ; . p. 2578.

4. Bauer SB. Special considerations of the overactive bladder in children. 60. Urology; 2002. p. 43.

5. Yousefichaijan P, Safi F, Rafiei M, Taherahmadi H, Fatahibayat G, Naziri M. Prenatal Risk Factors for Infantile Reflux Nephropathy.J Ped Nephrol. 2015;3(4):135-8.

6. Yousefichaijan P ; Rahmati SH ; Mohammadbeigi A ; Rajbaran M. . A. Clinical signs, causes, and risk factors of pediatric chronic kidney diseases. Inter J Pediatrics. 2016;4(6):1966-73.

7. Sokal R, Tata LJ, Fleming KM. Sex prevalence of major congenital anomalies in the United Kingdom: a national population-based study and international comparison meta-analysis. Birth Defects Res A Clin Mol Teratol. 2014;100(2):79-91. doi: 10.1002/bdra.23218. [PubMed: 24523198].

8. Tain $\mathrm{YL}$, Luh $\mathrm{H}$, Lin $\mathrm{CY}$, Hsu CN. Incidence and Risks of Congenital Anomalies of Kidney and Urinary Tract in Newborns: A Population-Based Case-Control Study in Taiwan. Medicine (Baltimore). 2016;95(5):e2659. doi: 10.1097/MD.0000000000002659. [PubMed: 26844492]. 
9. Raisanen S, Sankilampi U, Gissler M, Kramer MR, Hakulinen-Viitanen T, Saari J, et al. Smoking cessation in the first trimester reduces most obstetric risks, but not the risks of major congenital anomalies and admission to neonatal care: a population-based cohort study of 1,164,953 singleton pregnancies in Finland. J Epidemiol Community Health. 2014;68(2):159-64. doi:10.1136/jech-2013-202991. [PubMed: 24158704].

10. Sadiq S, Faiq SM, Idrees MK. Lumbosacral dysraphism as cause of neu- rogenic bladder: Magnetic Resonance Imaging based study from SIUT Pakistan. J Pak Med Assoc. 2015;65(5):501-5. [PubMed: 26028384].

11. Hsu CW, Yamamoto KT, Henry RK, De Roos AJ, Flynn JT. Prenatal risk factors for childhood CKD. J Am Soc Nephrol. 2014;25(9):2105-11. doi 10.1681/ASN.2013060582. [PubMed: 24744441].

12. Bauer SB. Neurogenic bladder: etiology and assessment. Pediatr Nephrol. 2008;23(4):541-51. doi:10.1007/s00467-008-0764-7. [PubMed: 18270749]. 